\title{
Micellization of a Cationic Surfactant in Mixed Aqueous and Non-aqueous Solvent Systems
}

\section{${ }^{* 1}$ GRACE, AGBIZU COOKEY 2DANIEL, OZIOMA UZOMA}

\author{
Department of Chemistry, Rivers State University of Science and Technology, Port Harcourt, Nigeria \\ Email; ${ }^{2}$ oziomah101@yahoo.com \\ Corresponding author:cookey.grace@ust.edu.ng
}

\begin{abstract}
The Micellisation behaviour of sodium dodecyl sulfate (SDS) in different mole fractions (0.1 0.5 and 0.9 ) of propylene glycol (PG) - water mixed solvents was investigated using electrical conductivity measurement at temperatures of 30,35 and $40{ }^{\circ} \mathrm{C}$. The critical micelle concentration (CMC) increased and the degree of counterion dissociation values $(\alpha)$ decreased with increase in the composition of PG. Variation of CMC with temperature is marked with U-shaped curves while the degree of counterion dissociation is linear with temperature. These results have been discussed based on the influence of PG on the cohesive forces of the mixed solvents as well as temperature effect on the thermal motion of mixed solvent molecules @JASEM
\end{abstract}

http://dx.doi.org/10.4314/jasem.v19i4.2

\section{INTRODUCTION}

A surfactant molecule is a surface-active agent with two distinct features; the polar head group and the non-polar alkyl chain part. Whereas the polar head group may or may not be charged, the non-polar tail usually comprises of hydrocarbon chains (Bidyut et al., 2009; Tyowua et al., 2012), though fluorocarbon units also exist. The dual molecular nature of surfactant molecules distinguishes them from other surface-active agents in that at low concentrations, they drastically reduce the unusual high surface tension of water through adsorption of the molecule at the air-water interface. Secondly and more significantly, surfactant molecules arrange themselves in bulk solutions into aggregates otherwise called micelles when the interface is fully saturated with the surfactant molecules. Micelle formation is driven by hydrophobic effect but opposed by electrostatic repulsion of the ionic head group (Jiang et al., 2009).

Cationic surfactants like sodium dodecyl sulphate, possess valuable characteristics like emulsification, wetting, water proofing, repellence, spreading, etc. which are relevant in several applications such as pharmaceuticals, enhanced oil recovery (Peter et al., 2008; Sansanwal, 2006; Srivastava, 2003), metallurgical process for ore concentration, and solubilization of water insoluble dyes (Nazar et al., 2010). A special group of cationic surfactants; quaternary ammonium surfactants is usually employed due to some inherent superior properties, including insensitivity to $\mathrm{pH}$ variations and high substantivity, encompassing the uptake of surfactant molecules from solution onto surfaces of negatively charged surfaces such as fibres, proteins, wools, metals, and pigments (Shaun and Vincent, 2009). Investigations about the micellization characteristics of different types of surfactants are often carried out mostly in water and in aqueous media containing additives that can alter the water structure (Fahimeh et al., 2000; Kabir-Ud-Din et al., 2006; Mehta et al., 2008; Akbas and Kartal, 2006; Niraula et al., 2013). The importance of studying micellization of surfactants in water-organic mixed-solvent systems is driven by both fundamental and practical considerations (Kabir-Ud-Din and Koya, 2010). In view of this, there has been a renewed interest on the study of adsorption and aggregation of surfactants in solvent media containing a binary mixture of water and a polar non-aqueous solvent in recent years (Sansanwal, 2006; Kabir-Ud-Din and Koya, 2010; Schgal et al., 2008; Ashish and Kallol, 2008). Despite extensive studies made on the micellization behaviour of surfactants in different types of media, it is still not exactly clear which property of a solvent controls micellization process. However, some authors have reported that high cohesive energies, dielectric constants, and considerable hydrogen bonding abilities between solvent molecules are prerequisite to aggregation of surfactants molecules in solution (Rodriguez et al., 2008).

Surfactants can only function effectively above a certain solution concentration called critical micelle 
concentration (CMC) i.e., the concentration above which they form aggregates or micelles. It is only above this concentration that significant changes in both physical and performance properties of surfactant solutions take place. CMC is a measure of the ability of a surfactant to form micelles. The lower the $\mathrm{CMC}$, the greater the ability and vice-versa. CMC can be obtained by measuring any surfactant solution property (e.g., surface tension, density, speed of sound, light scattering intensity, light absorption, molar conductivity, etc.) as a function of surfactant concentration [Nasiru et al. (2011) and Dominguez et al. (1997)] and an abrupt change of slope on the plot of the physical property versus surfactant concentration marks the CMC. For ionic surfactants, one of the frequently used physical properties has been electric conductivity. The plot of conductivity against the concentration of surfactant solution yields two straight lines above and below an inflection point. The intercept of these lines gives the CMC.

Addition of polar organic solvents to aqueous micellar solutions will alter the tendency of amphiphile molecules to avoid contact with water and hence the value of surfactant concentration at which micellization occurs. For this reason, it is important to investigate the role played by different non-aqueous solvents on the aggregation of surfactants. Thus, several studies have been carried out on the behaviour of surfactants in binary mixtures of water and organic solvents such as glycerol (Bakshi and Kaur, 1999), formamide (Desai et al., 2001), and ethylene glycol (Gharibi and Bloor, 1993). However, to the best of our knowledge, no studies have been done on the effect of propylene glycol additive on micelle formation of SDS in aqueous solution at the various temperatures. The results thereafter will give additional insight on the selection of mixed-solvent systems in the numerous industrial applications of SDS.

\section{MATERIALS AND METHODS}

Propylene glycol was of Analytical Grade and a product of Qualikems Chemicals, New Delhi. Sodium dodecyl Sulphate (99\% pure) was purchased from Lancaster Synthesis. All compounds were used without further purification.
The conductivity of SDS solutions in binary mixtures of water + propylene glycol was measured at different compositions of the organic solvents $(0.1$, 0.5 and 0.9$)$ and temperatures $\left(30,35\right.$ and $\left.40{ }^{\circ} \mathrm{C}\right)$. The binary mixture at each composition was used to prepare the solution of SDS at a concentration ten times its CMC (Cookey and Nwokobia, 2014A). The Conductivity measurements were carried out with a digital conductivity meter Model DDS-307A of cell constant $1.00 \mathrm{~cm}^{-1}$. The electrode was rinsed with distilled water after each measurement. For each measurement, $25 \mathrm{ml}$ of distilled water was first put in a beaker. A constant volume of the prepared SDS solution was successively added to the beaker using an Eppendorf pipette. For each addition, the solution was allowed to thermally equilibrate at the desired temperature for at least 10 minutes before the conductivity value was taken. In this way, the Precise Conductance measurements was $\pm 0.5 \%$. All experiments were done in a thermostated water-bath.

\section{RESULTS AND DISCUSSION}

Figure 1 shows the dependence of conductivity on concentration for solutions of SDS in binary mixed solvents of propylene glycol and water. The slope of the conductivity versus concentration curve increases before the brake point and decreases beyond it. The solution of an ionic surfactant behaves like a strong electrolyte (ions are free to move in solution) below the minimum concentration above which micelles are formed (CMC). Above the CMC, the solution is saturated with micelles or aggregates and the mobility of the ions is slowed down, thus the slope decreases. The value of the intercept of two straight lines below and above the inflection point gives the CMC (Cookey and Nwokobia, 2014B) of the solution at the given composition of propylene glycol. The ratio of the slope of the conductivity/concentration curve above and below the CMC is the degree of micelle ionization. 

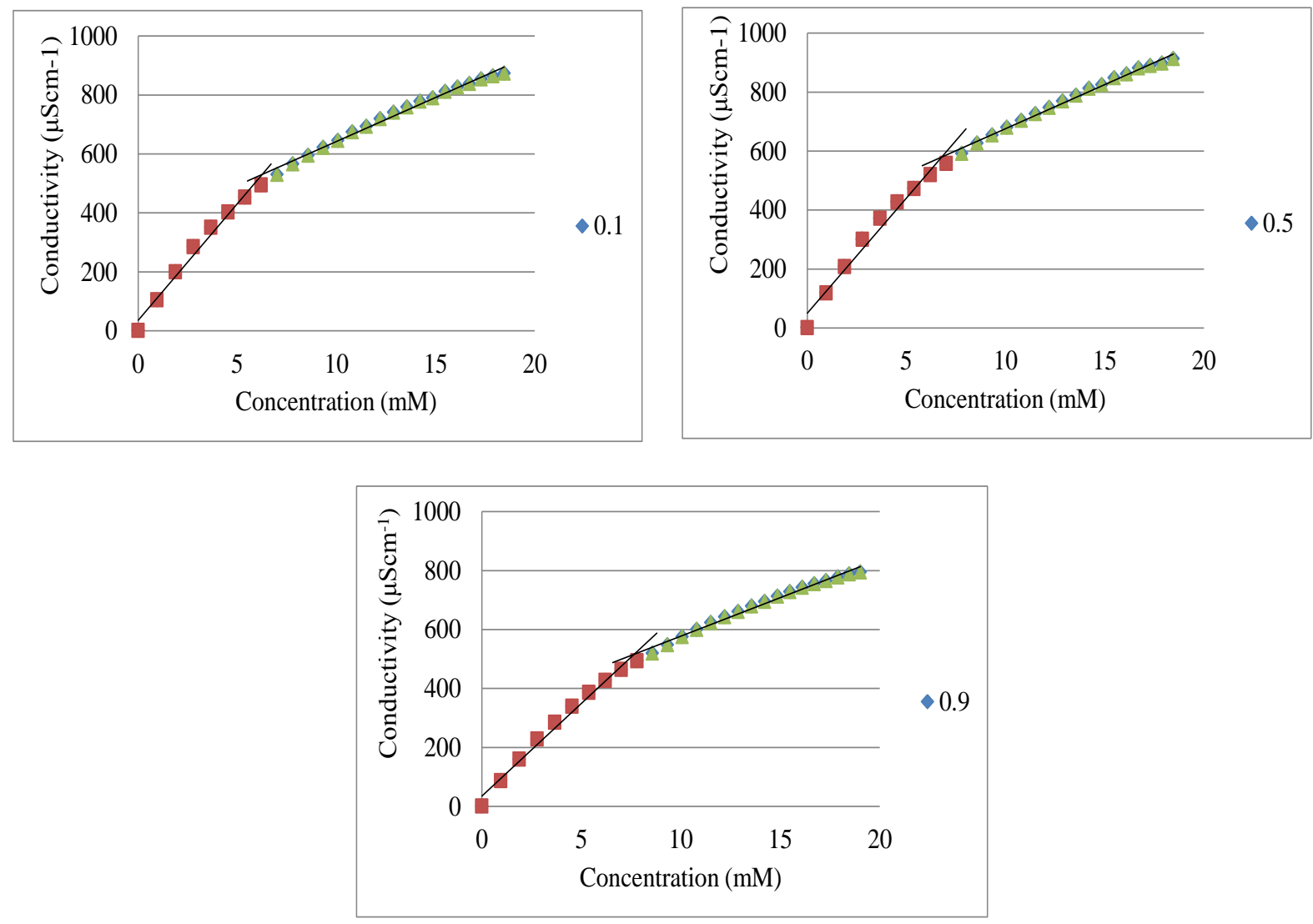

Fig 1: Conductivity versus concentration curves of solutions of SDS in binary mixtures of propylene glycol and water with intercepts of two straight lines below and above inflection points.

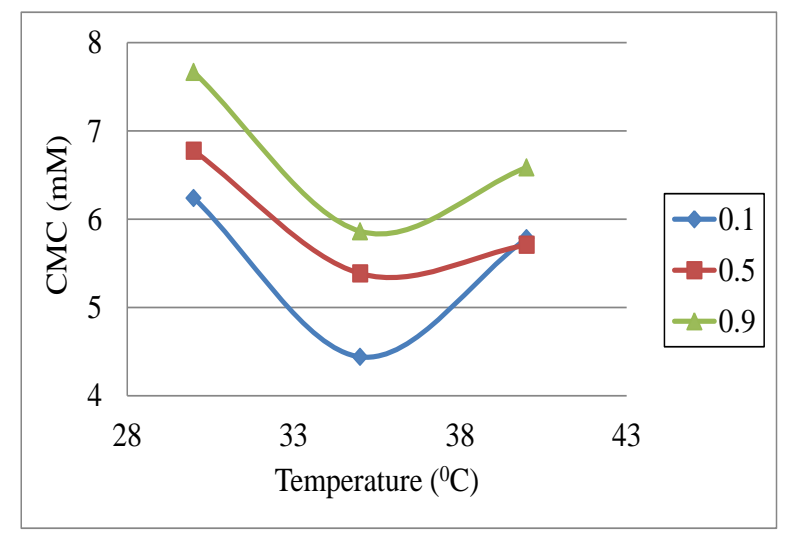

Fig. 2: CMC (mM) of solutions of SDS in binary mixtures of propylene glycol and water at different temperatures and compositions of propylene glycol.

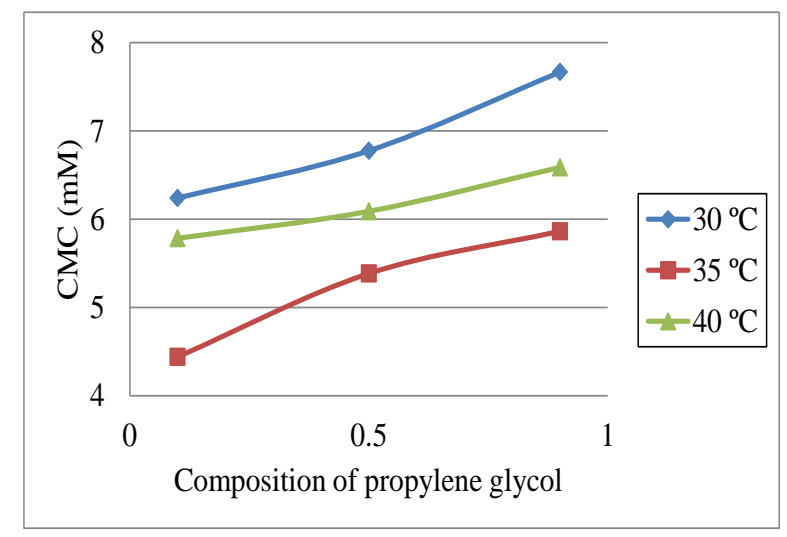

Fig 3: CMC (mM) of solutions of SDS in binary mixtures of propylene glycol and water at different temperatures and compositions of propylene glycol.
Figure 2 shows the plots of critical micelle concentration of SDS in binary mixtures of water and propylene glycol at varying compositions of propylene glycol. CMC is observed to increase with increasing compositions of propylene glycol implying that micelle formation in aqueous solution is disfavoured by the addition of propylene glycol. Similar observation has also been made by Adane in 2015 on the micellization of sodium dodecyl sulphate, hexadecyl trimethyl ammonium bromide and polyoxyethylene (20) sorbitan mono-oleate surfactants in binary mixed solvents of water $+1,2$ -

\section{${ }^{*}$ GRACE, AGBIZU COOKEY ${ }^{2}$ DANIEL, OZIOMA UZOMA}


ethanediol and water + 1,2,3-propanetriol at 298.15, 308.15 and 318.15 K. Prajapati and Patel (2012) have also reported the micellization of SDS, CTAB and tweens-80 but in Ethylene glycol/water and formamide/water mixed solvents using surface tension, viscosity and conductance measurements at $30^{\circ} \mathrm{C}$. Contrarily, it was observed that increase in the ratio of ethylene glycol lowered the $\mathrm{CMC}$ values of of SDS. As has been mentioned before, micelle formation is governed by a balance of two forces; the tendency of the hydrophilic head group to remain attracted to water and the hydrophobic tail to shield itself from water into the interior of the micelle. The ease with which this equilibrium is achieved determines the relative tendency with which micelles are formed and thus the value of CMC. Since increasing the composition of propylene glycol increases the value of the critical micelle concentration of SDS, it may imply that water is a better solvent than propylene glycol in terms of micellization of SDS. Recall that glycols are dihyroxy alcohols having two $\mathrm{OH}$ groups in one molecule. Thus propylene glycol has the ability to break up the structure of water molecules around the hydrophobic chains therefore decreasing the hydrophobic effect and resulting in increased values of CMC (Ghosh, 2009). However, increase in temperature increases the thermal motion of the water molecules which further disfavours the hydrophobic

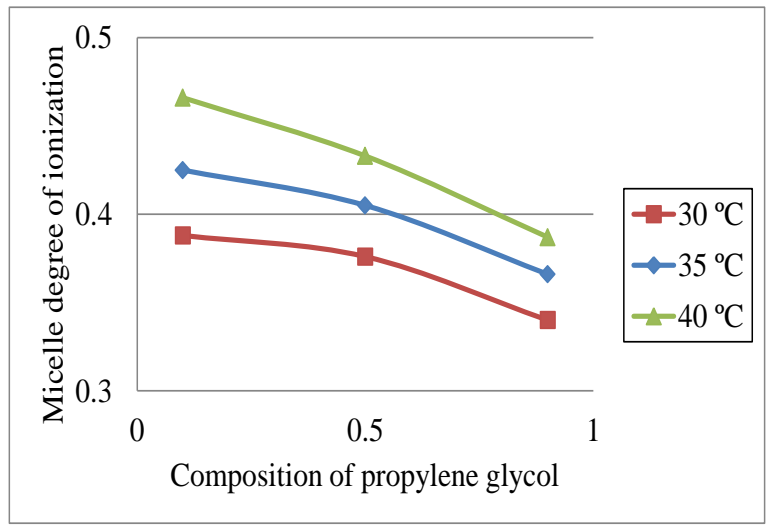

Fig 4: The degree of ionization of SDS micelles in binary mixtures of propylene glycol and water at different temperatures and compositions of propylene glycol.

The effect of propylene glycol on the degree of ionization of SDS micellar headgroup is shown in Figure 4. The decrease of the degree of dissociation of SDS micellar headgroup as the composition of propylene glycol increased is a reflection of the increasing critical micelle concentration of SDS with propylene glycol (Figure 2). This result may probably effect (Nagarajan and Wang 2000; MacIsaac et al., 2004) and thus micelle formation; therefore CMC values further increased.

The relationship between $\mathrm{CMC}$ and temperature is shown in Figure 2. CMC decreases as the temperature increases with a minimum at approximately $35{ }^{0} \mathrm{C}$ above which $\mathrm{CMC}$ increases again. The U-shaped CMC-temperature curve for organic solvent-water SDS solutions has previously been reported (Gholamreza et al., 2007; Kroflic et al., 2012). The minimum for CMC versus temperature curves occurring at approximately $35{ }^{0} \mathrm{C}$ has also been reported for aqueous solutions of some ionic surfactants such as decyltrimethyl ammonium chloride, dodecyltrimethyl ammonium chloride and tetradecyltrimethylammonium chloride respectively determined through conductivity measurements (Kroflic et al., 2012). The effect of temperature on micelle formation is usually attributed to two opposing factors (Aguiar et al., 2002); the degree of hydration of surfactant headgroups can be reduced, and so surfactant molecules spend less time interacting with the solvent molecules. This leads to faster aggregation, favouring micellization. Secondly, additives may disrupt the structure of water around the hydrophobic chain thus disfavouring micelle formation. Figure 3 illustrates that the first effect dominates at lower temperatures while the second comes to play at temperatures above $35{ }^{0} \mathrm{C}$

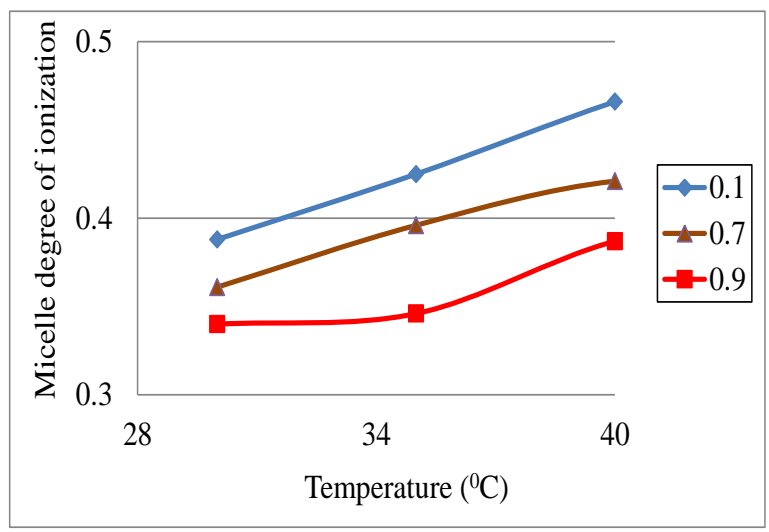

Fig 5: The degree of ionization of SDS micelles in binary mixtures of propylene glycol and water at different temperatures and compositions of propylene glycol.

be due to dilution effect. On the other hand, the increasing degree of counterion dissociation with temperature could be attributed to reduction in the aggregation number of SDS arising from a decrease in the charge density at the micellar surface. Similar observation has been made by Aguiar et al., 2002 on the thermodynamics and micellar properties of tetradecyl trimethyl ammonium bromide in

\section{${ }^{*}$ GRACE, AGBIZU COOKEY ${ }^{2}$ DANIEL, OZIOMA UZOMA}


formamide-water mixtures. Olaseni et al., 2012 have also reported that thermal forces disperse headgroups of ionic surfactants and so increase the distance between counterions and surfactant headgroups leading to increase in degree of ionization.

\section{CONCLUSION}

The micellization of sodium dodecyl sulphate in aqueous-propylene glycol binary mixtures has been investigated through conductivity measurements at different compositions of propylene glycol and temperatures. The critical micelle concentration of SDS increased while the degree of counterion dissociation decreased with increasing composition of the organic solvent. Variation of CMC with temperature is marked with U-shaped curves while the degree of counterion dissociation is linear with temperature.

\section{REFERENCE}

Aguiar, J; Molina-Bolivar, J A; Peula-Garcia, J M; Ruiz, C C (2002). Thermodynamics and micellar properties of tetradecyltrimethylammonium bromide in formamide-water mixtures. Journal of Colloid and Interface Science, 255, 382-390.

Akbas, H; Kartal, L (2006). Conductrimetric studies of hexadecyltrimethyl ammonium bromide in aqueous solution of ethanol and ethylene glycol. Colloid Journal, 68:125-130.

Ashish, S; Kallol, K G (2008). Micellization of cationic surfactant in binary aqueous solvents. Journal of surfactant detergent, 11, 28-298.

Bakshi, M S; Kaur, G (1999). Hexadecyl trimethyl ammonium bromide tetraacetyl trimethyl ammonium bromide mixed micelles in aqueous glycol oligomers. Journal of Macro Molecular Science, 36, 697-718.

Bidyut, S; Ghosh, S K; Basu, A; Paul, K K (2009). Micelle catalyzed oxidation of propan-2-ol to acetone by penta-valent vanadium in aqueous acid medium. Journal of molecular physics, 107, 615-619.

Cookey, G A; Nwokobia, F U (2014A). Effect of Ndecyl-n-n-dimethyl-3-ammonio-1-

propanesulfonate on the solution properties of sodium dodecyl sulfate. Journal of Applied Science and Environmental Management, 18, 523-527
Cookey, G A; Nwokobia, F U (2014B). Conductivity studies of binary mixtures of ionic and non-ionic surfactants atdifferent temperatures and concentrations. Journal of Applied Science and Environmental Management 18 530-534

Desai, P R; Jain, N J; Shairai, R K (2001). Energetics of drug-additive systems at the cloud point. Journal of Chemical and Engineering Data, 54, 387-391.

Dominguez, A; Fernandez, A; Gonzalez, N; Iglesias, E; Montenegro, L (1997). Determination of critical micelle concentration of some surfactants by three techniques. Journal of Chemical Education, 74; 1227-1231.

Fahimeh, J; Mojtaba, S; Naader, A (2000). Conductance study of the thermodynamics of micellization of I-hexadecylpyridinium bromide in (water co- solvent). Journal of chemical thermodynamics, 32, 755-765.

Fenta, A D (2005). Surface and thermodynamic studies of micellization of surfactants in binary mixtures of 1,2-ethanediol and 1,2,3-propanetriol with water. . International Journal of Physical Science, 10, 276-288.

Gharibi, H; Bloor, D M (1993). Electrochemical studies associated with the micellization of cationic surfactants in aqueous mixtures. Langmuir, 9, 110-112.

Gholamreza, D N; Mohammadreza, H; Bibi, S F B (2007). The effect of temperature on thermodynamic parameters of micellization of some surfactants. Journal of Applied Sciences, 7, 47-52.

Ghosh, D; Ghosh, K K (2009). Micellization behaviour of [C16-4-C16], 2Br- gemini surfactant in binary aqueous-solvent mixtures. Indian Journal of Chemistry, 48A, 1522-1526.

Jiang, N; Li, P; Wang, J; Yan, H; Thomas, R K (2005). Aggregation of hexadecyl trimethyl ammonium surfactants with various counterions in aqueous solution. Journal of colloid and interface science, 286, 755-760.

Kabir-Ud-Din; Koya, P A (2010). Effect of solvent media and temperature on the self-aggregation of cationic dimeric surfactant (14-6-14), studies of conductometric and fluorescence techniques. Langmuir, 26, 7905-7914.

\footnotetext{
${ }^{*}$ GRACE, AGBIZU COOKEY ${ }^{2}$ DANIEL, OZIOMA UZOMA
} 
Kabir-Ud-Din; Sidddiqui, U S; Kumar, S (2006), Micellization of monomeric and dimeric surfactants in polar non-aqueous -water -mixed solvents. Colloid Polymer Science, 284, 807-81

Kroflic, A; Sarac, B; Rogac, M B (2012). What affects the degree of micelle ionization: Conductivity study of alkyl trimethyl ammonium chlorides Acta Chimica. Slovenica, 59, 564-570.

MacIsaac, G; Al-Wardian, A; Glenn, K; Palepu R M (2004). Effects of di-, tri- and tetraethylene glycols on the thermodynamic and micellar properties of triton X-100 in water. Canadian Journal of Chemistry, 82, 1774-1780.

Mehta, S K; Savita, C; Bhasin, K K (2008). Selfassembly of cetylpyridinium chloride in waterDMF binary mixtures: A spectroscopic and physio chemical approach. Journal of Colloid and Interface Science, 321, 426-433.

Nagarajan, R; Wang, C-C (2000). Theory of surfactant aggregation in water/ethylene glycol mixed solvents. Langmuir, 16, 52425251.

Nasiru, T; Avila, L; Levine, M (2011). Determination of critical micelle concentration using UV spectroscopy. Journal of High School Research, $2,1-5$.

Nazar, M F; Shah, S S; Khosa, M A (2010). Interaction of azo dye with cationic surfactant under different $\mathrm{pH}$ conditions. Journal of surfactant detergent, 13, 529-536

Niraula, T P; Bhattarai, A; Chatterjee, S E (2013). Conductivity studies on the effect of $\mathrm{NaBr}$ on micellization of sodium dodecyl sulphate in pure water and methanol-water mixed solvent media at different temperatures. Journal of Modern trends in Science and Technology, 62-74.

Olaseni, S E; Oladoja, N A; Ololade, I. A; Aboluwoye, C. O; Osundiya, M O (2012). Micellization of cetyltrimethyl ammonium bromide in aqueous-organic media. Chemical Sciences Journal, 2012: CSJ, 52-63.
Peter, C G; Ian, A F; Thomas, T (2008). Aqueous solutions of transition metals containing micelles. Advance in colloid and interface science, 144, 13-23.

Prajapati, K; Patel, S (2012). Micellization of surfactants in mixed solvent of different polarity. Archives of Applied Science Research, 4, 662668.

Rodriguez, A; Gracini, M; Moya, M L (2008). Effects of addition of polar organic solvents on micellization. Langmuir, 24; 12785-12790.

Sansanwal, P K (2006). Effect of co-solute on the physio-chemical properties of surfactant solution. Journal of science and Industrial Research, 65, 57-66.

Shaun, C A; Vincent, S J C (2009). Adsorption of the cationic surfactant cetyltrimethylammonium bromide to silica in the presence of sodium salicylate, surface excess and kinetics. Langmuir, $25,13015-13026$

Schgal, P; Doc, H; Wimmer, R; Tanaka, R; Kosaka, $\mathrm{O}$ (2008). Mixed monolayer and micelle formation of cationic and zwitterionic surfactant of identical hydrocarbon tail in an aqueous medium; interfacial tension, fluorescence probe, dynamic light scattering, and viscosity studies. Journal of Dispersion Science and Technology, $29 ; 327-334$.

Srivastava, R C (2003). Liquid membrane phenomena, biological implication. Indian Journal of chemistry, 424, 2793-2799

Tyowua, A T; Yiase, S G; Wuanna, R A (2012). Manipulation of concentration-conductivity data of sodium dodecyl sulphate and sodium dodecyl benzene sulphonate in $\mathrm{KCl}$ solution in relation to micellization parameters. Chemical Science Journal, 79, 1-9. 